\title{
Free-Vibration Study of Thick Rectangular Plates using Polynomial Displacement Functions
}

\author{
I. C. Onyechere", O. M. Ibearugbulem, U. C. Anya, L. Anyaogu, C. T. G. Awodiji \\ Department of Civil Engineering, School of Engineering and Engineering Technology, Federal University of Technology, Owerri, Nigeria
}

DOI: $10.36348 /$ sjet.2020.v05i02.006 $\quad$ | Received: 06.02.2020 | Accepted: 16.02.2020 | Published: 23.02 .2020

*Corresponding author: Ignatius Chigozie Onyechere

\section{Abstract}

This paper applied polynomial displacement functions for free-vibration study of isotropic thick rectangular plates. The theory uses a third-order polynomial to describe how the transverse shear stress varies across the thickness of the plate and has no need for a shear correction factor. Polynomial expressions were used as the displacement functions ( $\mathrm{u}, \mathrm{v}$ and w) and also as shear deformation function $\mathrm{f}(\mathrm{z})$ in obtaining the general governing equations for the plate. For numerical illustrations, a thick rectangular plate whose four edges have simple supports (SSSS) was studied. The requirements for the edge conditions of the simply supported plate were satisfied and used in solving the general governing equation. This resulted to a linear equation used to generate for the plate, the non-dimensional fundamental natural frequency parameters for any value of span-thickness relation (a/t) and in-plane relation (b/a). To validate this study, results obtained herein were compared with related previous works in literature.

Keywords: Fundamental natural frequency, Shear deformation, Polynomial function, Displacement functions, plate, governing equation.

Copyright @ 2020: This is an open-access article distributed under the terms of the Creative Commons Attribution license which permits unrestricted use, distribution, and reproduction in any medium for non-commercial use (NonCommercial, or CC-BY-NC) provided the original author and source are credited.

\section{INTRODUCTION}

Plates have numerous applications in Engineering. Apart from static loads, time-dependent loads or dynamic loads could also act on plate structures. Thus, it is not adequate to design plate structures to withstand only static loads $[1,2]$. There are several number of discrete frequencies of the plate at which when time-dependent loads of equal frequencies act on the plate will result to large-amplitude vibrations, which could lead to structural failure. This phenomenum is known as resonance [3]. Thus, to rectify the above problem, it becomes necessary to conduct a free-vibration analysis of the rectangular plate so as to determine these frequencies that could cause resonance [1].

The Classical Plate Theory (CPT) did not consider the effects of the transverse shear strains and stresses and thus, restrict the model to thin plates [4]. Nevertheless, thick and moderately thick plates in which substantial transverse shear strains occur have numerous applications in engineering such as; bridge decks, cylinders for nuclear reactors, etc., The theory under-estimates of deflections and over-estimates the natural frequencies and buckling loads [5]. The first theory of beams that included the effects of shear deformation was developed by Timoshenko and this theory is now widely referred to as Timoshenko beam theory or First Order Shear Deformation Theory (FSDT) [6]. FSDT assumes a uniform shear strain distribution throughout the thickness of the plate. However, this assumption cannot be true and in order to correct this, a shear coefficient is introduced into the analysis [7]. As a result of these shortcomings of the (CPT) and (FSDT), the plate theories were further refined to circumvent the need for a shear correction factor and to get a convincing variation of the transverse shear strains within the plate's thickness. Such refined theories are called Higher Order Shear Deformation Theories (HSDT). Sayyad, I. I et al., [5] applied trigonometric shear deformation functions in their study on thick plates. Hashemi, S. H et al., [8] obtained exact characteristic equations for moderately thick plates using trigonometric displacement functions. Rajesh, K et al., [9] carried out vibration analysis of thick plates using trigonometric displacement functions. The present work applied a polynomial shear deformation function and polynomial displacement functions in free vibration analysis of isotropic thick rectangular plates with simple supports at the four edges. 


\section{Mathematical Formulations}

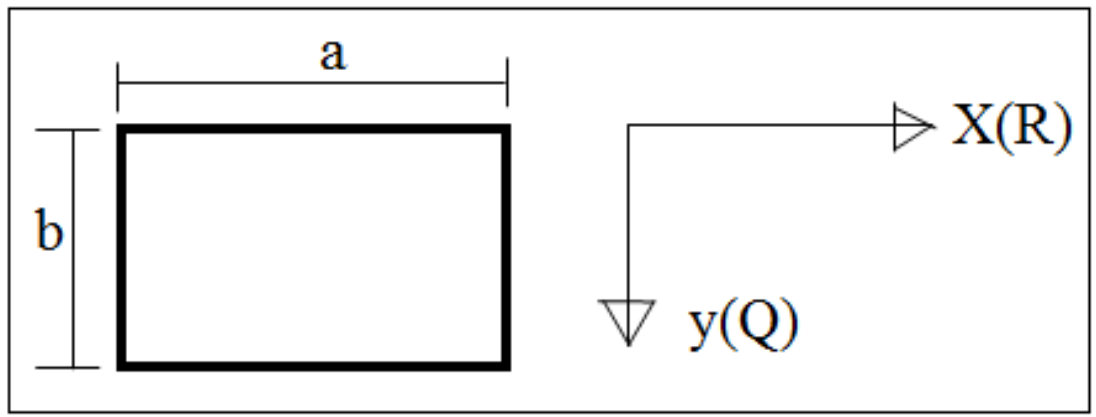

Fig-1: A Rectangular Plate

An isotropic rectangular thick plate of length ' $a$ ' in the horizontal axis, width ' $b$ ' in the vertical axis, and thickness ' $t$ ' in the transverse axis is shown in Fig-1.

The displacement functions of the present theory were derived in [10] as;

$$
\begin{aligned}
& u=-z \frac{\partial w}{\partial x}+f(z) \cdot \emptyset_{x} \ldots \ldots \ldots \ldots . . .(2.1) \\
& v=-z \frac{\partial w}{\partial y}+f(z) . \emptyset_{y} \ldots \ldots \ldots \ldots \ldots(2.2) \\
& w(x, y)=w_{x} \cdot w_{y}=\left(a_{0}+a_{1} R+a_{2} R^{2}+a_{3} R^{3}+a_{4} R^{4}\right) *\left(b_{0}+b_{1} Q+b_{2} Q^{2}+b_{3} Q^{3}+b_{4} Q^{4}\right)
\end{aligned}
$$

Where;

$\mathrm{u}, \mathrm{v}$ and $\mathrm{w}$ are the displacements in the $\mathrm{x}, \mathrm{y}$ and $\mathrm{z}$-directions respectively. $f(z)$ is the shear deformation function $\emptyset_{x}$ and $\emptyset_{y}$ are the shear rotations in $\mathrm{x}$ and $\mathrm{y}$-axis respectively given as;

$$
\emptyset_{x}=C_{x} \cdot \frac{\partial w}{\partial x}, \emptyset_{y}=C_{\mathrm{y}} \cdot \frac{\partial w}{\partial y}
$$

Where; $C_{x}, C_{y}, \mathrm{a}_{\mathrm{i}}$ and $\mathrm{b}_{\mathrm{i}}$ are constants.

$\mathrm{R}$ and $\mathrm{Q}$ are non-dimensional variables in $\mathrm{x}$ and $\mathrm{y}$-axis respectively given as;

$$
x=a R, y=b Q \ldots \ldots \ldots \ldots \ldots \ldots \ldots(2.3 \mathrm{c})
$$

Such that; $0 \leq x \leq a, 0 \leq R \leq 1,0 \leq y \leq b, 0 \leq Q \leq 1$

\section{Kinematics}

Using strain-displacement relationship of theory of elasticity, the shear strains are given as;

$$
\begin{aligned}
& \varepsilon_{x}=\frac{\mathrm{d} u}{d x}=-z \frac{\partial^{2} w}{\partial x^{2}}+f(z) \cdot \frac{\partial \emptyset_{x}}{\partial x} \ldots \ldots \ldots \ldots \ldots(2.4) \\
& \varepsilon_{y}=\frac{d v}{d y}=-z \frac{\partial^{2} w}{\partial y^{2}}+f(z) \cdot \frac{\partial \emptyset_{y}}{\partial y} \ldots \ldots \ldots \ldots \ldots \ldots(2.5) \\
& \gamma_{x y}=\frac{d u}{d y}+\frac{d v}{d x}=-2 z \frac{\partial^{2} w}{\partial x \partial y}+f(z) \cdot \frac{\partial \emptyset_{x}}{\partial y}+f(z) \cdot \frac{\partial \emptyset_{y}}{\partial x} \\
& \gamma_{x z}=\frac{d u}{d z}+\frac{d w}{d x}=f(z) \cdot \frac{\partial \emptyset_{x}}{\partial z} \ldots \ldots \ldots \ldots \ldots(2.7) \\
& \gamma_{y z}=\frac{d v}{d z}+\frac{d w}{d y}=f(z) \cdot \frac{\partial \emptyset_{y}}{\partial z} \ldots \ldots \ldots \ldots \ldots(2.8)
\end{aligned}
$$

The stress-strain relation of the isotropic plate can be written as follows;

$$
\begin{aligned}
& \sigma_{x}=\frac{E}{1-\mu^{2}}\left[\varepsilon_{x}+\mu \varepsilon_{y}\right] \\
& \sigma_{y}=\frac{E}{1-\mu^{2}}\left[\mu \varepsilon_{x}+\varepsilon_{y}\right] \\
& \tau_{x y}=\frac{E(1-\mu)}{2\left(1-\mu^{2}\right)} \gamma_{x y} \ldots \ldots .
\end{aligned}
$$




$$
\begin{aligned}
\tau_{x z} & =\frac{E(1-\mu)}{2\left(1-\mu^{2}\right)} \gamma_{x z} \\
\tau_{y z} & =\frac{E(1-\mu)}{2\left(1-\mu^{2}\right)} \gamma_{y z}
\end{aligned}
$$

$E$ and $\mu$ respectively represent the elastic modulus and the Poisson's ratio of the material.

\section{Strain Energy, $\Psi$.}

The Strain energy stored in a continuum of the thick plate under study is defined as;

$$
\Psi=\frac{1}{2} \int_{x} \int_{y}\left[\int_{-}^{t / 2}\left(\sigma_{x} \varepsilon_{x}+\sigma_{y} \varepsilon_{y}+\tau_{x y} \gamma_{x y}+\tau_{x z} \gamma_{x z}+\tau_{y z} \gamma_{y z}\right) d z\right] d x d y
$$

Let;

$$
\Psi_{1}=\sigma_{x} \varepsilon_{x}+\sigma_{y} \varepsilon_{y}+\tau_{x y} \gamma_{x y}+\tau_{x z} \gamma_{x z}+\tau_{y z} \gamma_{y z}
$$

Substituting Eqs. (2.5) - (2.13) into Eq. (2.14b) gives;

$$
\begin{aligned}
& \Psi_{1}=\frac{E}{1-\mu^{2}}\left[\left[z^{2}\left(\frac{\partial^{2} w}{\partial x^{2}}\right)^{2}-2 z f(z) \cdot \frac{\partial \emptyset_{x}}{\partial x}\left(\frac{\partial^{2} w}{\partial x^{2}}\right)+f^{2}(z) \cdot\left(\frac{\partial \emptyset_{x}}{\partial x}\right)^{2}\right]+\left[2 z^{2}\left(\frac{\partial^{2} w}{\partial x \partial y}\right)^{2}-2 Z f(z) \cdot \frac{\partial \emptyset_{x}}{\partial y} \frac{\partial^{2} w}{\partial x \partial y}-\right.\right. \\
& \left.2 Z f(z) \cdot \frac{\partial \emptyset_{y}}{\partial x} \frac{\partial^{2} w}{\partial x \partial y}\right]+\left[z^{2}\left(\frac{\partial^{2} w}{\partial y^{2}}\right)^{2}-2 z f(z) \cdot \frac{\partial \emptyset_{y}}{\partial y} \frac{\partial^{2} w}{\partial y^{2}}+f^{2}(z)\left(\frac{\partial \emptyset_{y}}{\partial y}\right)^{2}\right]+(1+\mu)\left[f^{2}(z) \cdot \frac{\partial \emptyset_{y}}{\partial x} \frac{\partial \emptyset_{x}}{\partial y}\right]+ \\
& \left.\frac{(1-\mu)}{2}\left[f^{2}(z)\left(\frac{\partial \emptyset_{x}}{\partial y}\right)^{2}+f^{2}(z)\left(\frac{\partial \emptyset_{y}}{\partial x}\right)^{2}\right]+\frac{(1-\mu)}{2} \cdot\left(\frac{d f(z)}{d z}\right)^{2} \cdot\left[\emptyset_{x}^{2}+\emptyset_{y}^{2}\right]\right] \ldots \ldots \ldots(2.15)
\end{aligned}
$$

Let;

$$
\begin{aligned}
& \Gamma=\int_{-t / 2}^{t / 2} z^{2} d z=\frac{t^{3}}{12}, \Gamma H_{1}=\int_{-t / 2}^{t / 2} z^{2} d z, \Gamma H_{2}=\int_{-t / 2}^{t / 2}[z f(z)] d z, \Gamma H_{3}=\int_{-t / 2}^{t / 2}\left[(f(z))^{2}\right] d z, \Gamma \frac{\alpha^{2}}{a^{2}} H_{4}= \\
& \left.\int_{-t / 2}^{t / 2}\left[\frac{d f(z)}{d z}\right]^{2} d z, D=\frac{\Gamma E}{1-\mu^{2}}=\frac{E t^{3}}{2\left(1-\mu^{2}\right)} \ldots \ldots \ldots \ldots \ldots . . .12 .16\right)
\end{aligned}
$$

The shear deformation function $f(z)$ used in the present study is a third order polynomial function derived in [10] and presented here as;

$$
f(z)=z-\frac{7 z^{3}}{5 t^{2}}
$$

Thus, from Eq. (2.17), Eqs. (2.18a) - (2.18e) are obtained;

$$
\begin{aligned}
& \int_{-t / 2}^{t / 2}\left(z^{2}\right) d z=\left[\frac{z^{3}}{3}\right]_{-t / 2}^{t / 2}=\left(\frac{1}{3}\right)\left[\frac{t^{3}}{8}--\frac{t^{3}}{8}\right]=2\left(\frac{1}{3}\right) \cdot\left(\frac{t^{3}}{8}\right)=\frac{t^{3}}{12} \\
& (f(z))^{2}=z^{2}-\frac{14 z^{4}}{5 t^{2}}+\frac{49 z^{6}}{25 t^{4}}, \int_{-\frac{t}{2}}^{\frac{t}{2}}(f(z))^{2} d z=\left[\frac{z^{3}}{3}-\frac{14 z^{5}}{25 t^{2}}+\frac{7 z^{7}}{25 h^{4}}\right]_{-\frac{t}{2}}^{\frac{t}{2}}=\frac{253 t^{3}}{4800} \\
& z f(z)=z^{2}-\frac{7 z^{4}}{5 t^{2}}, \int_{-\frac{t}{2}}^{\frac{t}{2}} z f(z) d z=\frac{t^{3}}{12}-\frac{7 t^{3}}{400}=\frac{79 t^{3}}{1200} \\
& \left(\frac{d f(z)}{d z}\right)^{2}=1-\frac{42 z^{2}}{5 t^{2}}+\frac{441 z^{4}}{25 t^{4}}, \int_{-\frac{t}{2}}^{\frac{t}{2}}\left(\frac{d f(z)}{d z}\right)^{2} d z=\left[z-\frac{14 z^{3}}{5 t^{2}}+\frac{441 z^{5}}{125 t^{4}}\right]_{-\frac{t}{2}}^{\frac{t}{2}}=\frac{1041 t}{2000}
\end{aligned}
$$

Substituting Eq. (2.18a - 2.18d) into Eq. (2.16), Eq. (2.19a) - (2.19d) were obtained.

$$
H_{1}=1, H_{2}=0.79, H_{3}=0.6325, H_{4}=6.246
$$

$\mathrm{D}$ is the flexural rigidity of the plate, and $\propto=a / t$ is the span-depth ratio. 
Substituting Eq. (2.16) into Eq. (2.15) and integrating yields;

$$
\begin{aligned}
& \int_{-\frac{t}{2}}^{\frac{t}{2}}\left(\Psi_{1}\right) d z= \\
& \frac{\boldsymbol{E} \Gamma}{\mathbf{1}-\mu^{2}}\left[\left[H_{1}\left(\frac{\partial^{2} w}{\partial x^{2}}\right)^{2}-2 H_{2} \cdot \frac{\partial \emptyset_{x}}{\partial x}\left(\frac{\partial^{2} w}{\partial x^{2}}\right)+H_{3} \cdot\left(\frac{\partial \emptyset_{x}}{\partial x}\right)^{2}\right]+\left[2 H_{1}\left(\frac{\partial^{2} w}{\partial x \partial y}\right)^{2}-2 H_{2} \cdot \frac{\partial \emptyset_{x}}{\partial y} \frac{\partial^{2} w}{\partial x \partial y}-2 H_{2} \cdot \frac{\partial \emptyset_{y}}{\partial x} \frac{\partial^{2} w}{\partial x \partial y}\right]+\right. \\
& {\left[H_{1}\left(\frac{\partial^{2} w}{\partial y^{2}}\right)^{2}-2 H_{2} \frac{\partial \emptyset_{y}}{\partial y} \frac{\partial^{2} w}{\partial y^{2}}+H_{3}\left(\frac{\partial \emptyset_{y}}{\partial y}\right)^{2}\right]+(1+\mu)\left[H_{3} \cdot \frac{\partial \emptyset_{y}}{\partial x} \frac{\partial \emptyset_{x}}{\partial y}\right]+\frac{(1-\mu)}{2}\left[H_{3}\left(\frac{\partial \emptyset_{x}}{\partial y}\right)^{2}+H_{3}\left(\frac{\partial \emptyset_{y}}{\partial x}\right)^{2}\right]+} \\
& \left.\frac{(1-\mu)}{2} \cdot \frac{\alpha^{2}}{a^{2}} H_{4}\left[\emptyset_{x}{ }^{2}+\emptyset_{y}{ }^{2}\right]\right] \ldots \ldots \ldots \ldots(2.20) \\
& \boldsymbol{\Psi}= \\
& \frac{\mathbf{1}}{2} \int_{\boldsymbol{x}} \int_{\boldsymbol{y}}\left[\int_{-t / 2}^{t}\left(\boldsymbol{\Psi}_{1}\right) \boldsymbol{d z}\right] \boldsymbol{d x} \boldsymbol{d y}=\frac{D}{2} \int_{x} \int_{y}\left[\left[H_{1}\left(\frac{\partial^{2} w}{\partial x^{2}}\right)^{2}-2 H_{2} \cdot \frac{\partial \emptyset_{x}}{\partial x}\left(\frac{\partial^{2} w}{\partial x^{2}}\right)+H_{3} \cdot\left(\frac{\partial \emptyset_{x}}{\partial x}\right)^{2}\right]+\left[2 H_{1}\left(\frac{\partial^{2} w}{\partial x \partial y}\right)^{2}-\right.\right. \\
& \left.2 H_{2} \cdot \frac{\partial \emptyset_{x}}{y} \frac{\partial^{2} w}{\partial x \partial y}-2 H_{2} \cdot \frac{\partial \emptyset_{y}}{\partial x} \frac{\partial^{2} w}{\partial x \partial y}\right]+\left[H_{1}\left(\frac{\partial^{2} w}{\partial y^{2}}\right)^{2}-2 H_{2} \frac{\partial \emptyset_{y}}{\partial y} \frac{\partial^{2} w}{\partial y^{2}}+H_{3}\left(\frac{\partial \emptyset_{y}}{\partial y}\right)^{2}\right]+(1+\mu)\left[H_{3} \cdot \frac{\partial \emptyset_{y}}{\partial x} \frac{\partial \emptyset_{x}}{\partial y}\right]+ \\
& \left.\frac{(1-\mu)}{2}\left[H_{3}\left(\frac{\partial \emptyset_{x}}{\partial y}\right)^{2}+H_{3}\left(\frac{\partial \emptyset_{y}}{\partial x}\right)^{2}\right]+\frac{(1-\mu)}{2} \cdot \frac{\alpha^{2}}{a^{2}} H_{4} \cdot\left[\emptyset_{x}{ }^{2}+\emptyset_{y}{ }^{2}\right]\right] d x d y \ldots \ldots \ldots \ldots \ldots . .(2.21)
\end{aligned}
$$

The average total work performed by perturbation on the plate is given by;

$$
\Lambda=-\frac{m}{2} \cdot \lambda^{2} \int_{\mathrm{x}} \int_{\mathrm{y}}\left(w^{2}\right) \partial x \partial y
$$

\section{Total Potential Energy}

Total potential energy ' $\mathcal{G}$ ', of the thick plate is the sum of strain energy, $\boldsymbol{\Psi}$ and external work, $\Lambda$.

$$
\begin{aligned}
& \mathcal{G}=\boldsymbol{\Psi}+\Lambda=\frac{D}{2} \int_{x} \int_{y}\left[\left[H_{1}\left(\frac{\partial^{2} w}{\partial x^{2}}\right)^{2}-2 H_{2} \cdot \frac{\partial \emptyset_{x}}{\partial x}\left(\frac{\partial^{2} w}{\partial x^{2}}\right)+H_{3} \cdot\left(\frac{\partial \emptyset_{x}}{\partial x}\right)^{2}\right]+\left[2 H_{1}\left(\frac{\partial^{2} w}{\partial x \partial y}\right)^{2}-2 H_{2} \cdot \frac{\partial \emptyset_{x}}{\partial y} \frac{\partial^{2} w}{\partial x \partial y}-\right.\right. \\
& \left.2 H_{2} \cdot \frac{\partial \emptyset_{y}}{\partial x} \frac{\partial^{2} w}{\partial x \partial y}\right]+\left[H_{1}\left(\frac{\partial^{2} w}{\partial y^{2}}\right)^{2}-2 H_{2} \frac{\partial \emptyset_{y}}{\partial y} \frac{\partial^{2} w}{\partial y^{2}}+H_{3}\left(\frac{\partial \emptyset_{y}}{\partial y}\right)^{2}\right]+(1+\mu)\left[H_{3} \cdot \frac{\partial \emptyset_{y}}{\partial x} \frac{\partial \emptyset_{x}}{\partial y}\right]+\frac{(1-\mu)}{2}\left[H_{3}\left(\frac{\partial \emptyset_{x}}{\partial y}\right)^{2}+\right. \\
& \left.\left.H_{3}\left(\frac{\partial \emptyset_{y}}{\partial x}\right)^{2}\right]+\frac{(1-\mu)}{2} \cdot \frac{\alpha^{2}}{a^{2}} H_{4}\left[\emptyset_{x}^{2}+\emptyset_{y}^{2}\right]\right] d x d y-\frac{m}{2} \cdot \lambda^{2} \int_{\mathrm{x}} \int_{\mathrm{y}}\left(w^{2}\right) \partial x \partial y \ldots
\end{aligned}
$$

Let;

$$
w=J_{1} h \ldots \ldots \ldots \ldots \ldots(2.24 a)
$$

Substituting Eq. (2.24a) into Eq. (2.3b) gives;

$$
\emptyset_{x}=C_{\mathrm{a}} \cdot J_{1} \frac{\partial h}{\partial x}=J_{2} \frac{\partial h}{\partial x}, \emptyset_{y}=C_{\mathrm{b}} \cdot J_{1} \frac{\partial h}{\partial y}=J_{3} \frac{\partial h}{\partial y}
$$

Where; $J_{2}=C_{a} . J_{1}, J_{3}=C_{\mathrm{b}} . J_{1}, J_{1}, J_{2}, J_{3}$ are constants.

Substituting Eq. (2.24a) and (2.24b) into Eq. (2.23) and multiplying each term by $\frac{a^{4}}{a^{4}}$ gives;

$$
\begin{aligned}
& \mathcal{G}=\frac{D a b}{2 a^{4}} \int_{0}^{1} \int_{0}^{1}\left[\left(J_{1}{ }^{2} H_{1}-2 J_{1} J_{2} H_{2}+J_{2}{ }^{2} H_{3}\right)\left(\frac{d^{2} h}{d R^{2}}\right)^{2}+\left(J_{1}{ }^{2} H_{1}-2 J_{1} J_{3} H_{2}+J_{3}{ }^{2} H_{3}\right) \frac{1}{P^{4}}\left(\frac{d^{2} h}{d Q^{2}}\right)^{2}+\left(2 J_{1}{ }^{2} H_{1}-\right.\right. \\
& \left.2 J_{1} J_{2} H_{2}-2 J_{1} J_{3} H_{2}\right) \cdot \frac{1}{P^{2}}\left(\frac{d^{2} h}{d R^{2}} \cdot \frac{d^{2} h}{d Q^{2}}\right)+(1+\mu) J_{2} J_{3} H_{3} \cdot \frac{1}{P^{2}}\left(\frac{d^{2} h}{d R^{2}} \cdot \frac{d^{2} h}{d Q^{2}}\right)+\left[\left(\frac{1-\mu}{2}\right)\left(J_{2}{ }^{2} H_{3}+J_{3}{ }^{2} H_{3}\right)\right] \cdot \frac{1}{P^{2}}\left(\frac{d^{2} h}{d R^{2}} \cdot \frac{d^{2} h}{d Q^{2}}\right)+ \\
& \left.\left.\propto^{2}\left(\frac{1-\mu}{2}\right) \cdot\left(J_{2}{ }^{2} H_{4}\right)\left(\frac{d h}{d R}\right)^{2}+\frac{\alpha^{2}}{P^{2}}\left(\frac{1-\mu}{2}\right)\left(J_{3}{ }^{2} H_{4}\right)\left(\frac{d h}{d Q}\right)^{2}\right] d R d Q-\frac{a b J_{1}}{2} \int_{0}^{1} \int_{0}^{1}\left[m \lambda^{2}\left(h^{2}\right)\right] \partial R \partial Q \ldots \ldots \ldots \ldots \ldots . . . . . . . .25\right)
\end{aligned}
$$

Where the aspect ratio $\mathrm{P}$, is given as; $\mathrm{P}=\mathrm{b} / \mathrm{a}$.

\section{General Governing Equation}

Adopting Ritz method by minimizing the total energy equation, we obtain;

$$
\frac{d \mathcal{G}}{d J_{1}}=0, \frac{d \mathcal{G}}{d J_{2}}=0, \frac{d \mathcal{G}}{d J_{3}}=0
$$

Evaluating Eq. (2.26), Eq. (2.27a) - (2.27c) are obtained. 


$$
\begin{aligned}
& \frac{D}{a^{4}}\left[\left(T_{1} H_{1}+\frac{T_{3}}{P^{4}} H_{1}+\frac{2 T_{2}}{P^{2}} H_{1}\right) J_{1}+\left(-T_{1} H_{2}+\frac{T_{2}}{P^{2}} H_{2}\right) J_{2}+\left(-\frac{T_{3}}{P^{4}} H_{2}-\frac{T_{2}}{P^{2}} H_{2}\right) J_{3}\right]=\left(m \lambda^{2} T_{6}\right) J_{1} \ldots . \\
& \frac{D}{a^{4}}\left[\left(-T_{1} H_{2}-\frac{T_{2}}{P^{2}} H_{2}\right) J_{1}+\left(T_{1} H_{3}+\left(\frac{1-\mu}{2 P^{2}}\right) T_{2} H_{3}+\left(\frac{1-\mu}{2}\right) \propto^{2} T_{4} H_{4}\right) J_{2}+\left(\left(\frac{1+\mu}{2 P^{2}}\right) T_{2} H_{3}\right) J_{3}\right]=0 . \\
& \frac{D}{a^{4}}\left[\left(-\frac{T_{3}}{P^{4}} H_{2}-\frac{T_{2}}{P^{2}} H_{2}\right) J_{1}+\left(\left(\frac{1+\mu}{2 P^{2}}\right) T_{2} H_{3}\right) J_{2}+\left(\frac{T_{3}}{P^{4}} H_{3}+\left(\frac{1-\mu}{2 P^{2}}\right) T_{2} H_{3}+\left(\frac{1-\mu}{2 P^{2}}\right) \propto^{2} T_{5} H_{4}\right) J_{3}\right]=0
\end{aligned}
$$

Where;

$$
\begin{aligned}
& T_{1}=\int_{0}^{1} \int_{0}^{1}\left(\frac{\partial^{2} h}{\partial R^{2}}\right)^{2} \partial R \partial Q, T_{2}=\int_{0}^{1} \int_{0}^{1}\left(\frac{\partial^{2} h}{\partial R^{2}} \cdot \frac{\partial^{2} h}{\partial Q^{2}}\right) \partial R \partial Q, T_{3}=\int_{0}^{1} \int_{0}^{1}\left(\frac{\partial^{2} h}{\partial Q^{2}}\right)^{2} \partial R \partial Q, T_{4}=\int_{0}^{1} \int_{0}^{1}\left(\frac{\partial \mathrm{h}}{\partial \mathrm{R}}\right)^{2} \partial R \partial Q, T_{5}= \\
& \left.\int_{0}^{1} \int_{0}^{1}\left(\frac{\partial \mathrm{h}}{\partial \mathrm{Q}}\right)^{2} \partial R \partial Q, T_{6}=\int_{0}^{1} \int_{0}^{1}(h)^{2} \partial R \partial Q \ldots \ldots \ldots \ldots . . .2 .28\right)
\end{aligned}
$$

Re-writing Eq. (2.27a) - (2.27c) in matrix form, yields Eq. (2.29)

$$
\left[\begin{array}{lll}
L_{11} & L_{12} & L_{13} \\
L_{21} & L_{22} & L_{23} \\
L_{31} & L_{32} & L_{33}
\end{array}\right]\left\{\begin{array}{l}
J_{1} \\
J_{2} \\
J_{3}
\end{array}\right\}=\frac{a^{4}}{D}\left[\begin{array}{c}
m \lambda^{2} T_{6} \\
0 \\
0
\end{array}\right]\left\{\begin{array}{l}
J_{1} \\
J_{2} \\
J_{3}
\end{array}\right\} \ldots
$$

Eq. (2.29) is the general governing Equation for thick rectangular plates of arbitrary boundary conditions.

$$
\begin{aligned}
& L_{11}=H_{1}\left(T_{1}+\frac{2 T_{2}}{P^{2}}+\frac{T_{3}}{P^{4}}\right), L_{12}=-H_{2}\left(T_{1}+\frac{T_{2}}{P^{2}}\right), L_{13}=-H_{2}\left(\frac{T_{2}}{P^{2}}+\frac{T_{3}}{P^{4}}\right), L_{21}=L_{12}, L_{22}=T_{1} H_{3}+\left(\frac{1-\mu}{2 P^{2}}\right) T_{2} H_{3}+ \\
& \left(\frac{1-\mu}{2}\right) \propto^{2} K_{4} H_{4}, L_{23}=\left(\frac{1+\mu}{2 P^{2}}\right) K_{2} H_{3}, L_{31}=L_{13}, L_{32}=L_{23}, L_{33}=\left(\frac{1-\mu}{2 P^{2}}\right) T_{2} H_{3}+\frac{T_{3}}{P^{4}} H_{3}+\left(\frac{1-\mu}{2 P^{2}}\right) \propto^{2} T_{5} H_{4} \\
& \ldots \ldots \ldots \ldots \ldots \ldots \ldots . . . . . .30)
\end{aligned}
$$

\section{Analytical Equation for Free-Vibration Analysis}

The general governing equation derived and presented in Eq. (2.29) can be written as;

$$
\left[\begin{array}{lll}
S_{11} & S_{12} & S_{13} \\
S_{21} & S_{22} & S_{23} \\
S_{31} & S_{32} & S_{33}
\end{array}\right]\left[\begin{array}{l}
J_{1} \\
J_{2} \\
J_{3}
\end{array}\right\}=\frac{m a^{4} \lambda^{2}}{D}\left[\begin{array}{l}
1 \\
0 \\
0
\end{array}\right]\left(\begin{array}{c}
J_{1} \\
J_{2} \\
J_{3}
\end{array}\right\}
$$

Where;

$$
S_{i j}=L_{i j} * 1 / T_{6}
$$

Solving Eq. (3.1) using substitution method yields;

$$
J_{2}=\left[\frac{-S_{23} \cdot S_{31}+S_{33} \cdot S_{21}}{S_{32}{ }^{2}-S_{33} \cdot S_{22}}\right] J_{1}, J_{3}=\left[\frac{-S_{23} \cdot S_{21}+S_{22} \cdot S_{31}}{S_{32}{ }^{2}-S_{33} \cdot S_{22}}\right] J_{1}
$$

Substituting Eq. (3.3) into the first equation line in the matrix given in Eq. (3.1) gives;

$$
S_{11}+S_{12} \cdot\left[\frac{-S_{23} \cdot S_{31}+S_{33} \cdot S_{21}}{S_{32}{ }^{2}-S_{33} \cdot S_{22}}\right]+S_{13} \cdot\left[\frac{-S_{23} \cdot S_{21}+S_{22} \cdot S_{31}}{S_{32}{ }^{2}-S_{33} \cdot S_{22}}\right]=\frac{m a^{4} \lambda^{2}}{D}=\Delta^{2}
$$

Where $\Delta^{2}$ is a non - dimensional natural frequency parameter given as;

$$
\Delta^{2}=\frac{m a^{4} \lambda^{2}}{D}
$$

\section{Boundary Conditions}

The general Polynomial Deflection Function ' $w$ ' for thick plates is given in Eq. (2.3a). A close look at Eq. (2.3a) shows that it is a product of two orthogonal beams; one in $x(R)$-axis and the other in the $y(Q)$-axis. The deflection Equations for the two beams are given as Eqs. (3.6a) and (3.6b) respectively.

$$
\begin{aligned}
& w_{x}=\left(a_{0}+a_{1} R+a_{2} R^{2}+a_{3} R^{3}+a_{4} R^{4}\right) . \\
& w_{y}=\left(b_{0}+b_{1} Q+b_{2} Q^{2}+b_{3} Q^{3}+b_{4} Q^{4}\right)
\end{aligned}
$$

For the beam simply supported at both edges, the boundary conditions are as;

$$
\text { (i) At } R=0 ; w_{x}=0, \frac{\partial^{2} w_{x}}{\partial R^{2}}=0 \text { (ii) At } R=1 ; w_{x}=0, \frac{\partial^{2} w_{x}}{\partial R^{2}}=0 \text {. }
$$

Upon satisfaction of the conditions in Eq. (3.7), the following equation is obtained;

$$
\begin{aligned}
& w=w_{x} \cdot w_{y}=a_{4}\left(R-2 R^{3}+R^{4}\right) \cdot b_{4}\left(Q-2 Q^{3}+Q^{4}\right) \\
& w=J_{1} h=J_{1}\left(R-2 R^{3}+R^{4}\right) .\left(Q-2 Q^{3}+Q^{4}\right) \ldots \ldots
\end{aligned}
$$


Where;

$$
J_{1}=a_{4} b_{4}, h=\left(R-2 R^{3}+R^{4}\right) \cdot\left(Q-2 Q^{3}+Q^{4}\right)
$$

$\mathrm{H}$ is the shape function for the SSSS thick plate, $J_{1}$ is the amplitude.

Substituting Eq. (3.10) into Eq. (2.28) gives;

$$
T_{1}=0.23619 ; T_{2}=0.23592 ; T_{3}=0.23619 ; T_{4}=0.0239 ; T_{5}=0.0239
$$

\section{NUMERICAL RESULTS AND DISCUSSION}

Eq. (3.4) is a simple linear equation used to generate the fundamental natural frequency parameters for the plate at different aspect ratios, $\mathrm{P}=\mathrm{b} / \mathrm{a}$ and different span-depth ratios $(\mathrm{a} / \mathrm{t})$. Eq. (3.11) provides the stiffness ' $T_{i}$ ' for SSSS thick plate. The values of the non-dimensional fundamental natural frequency parameter $\Delta$ at different values of span-depth ratios and aspect ratios (b/a) generated from Eq. (3.4) are presented on Table-1. Tables 2 and 3 show the comparison of the results of the present study with the results of previous scholars. The graphs of the nondimensional fundamental natural frequency parameter $\Delta$ against the span-depth ratio of SSSS thick rectangular plate for the present study and previous researchers are presented in Figs 1 and 2.

\begin{tabular}{|c|c|c|c|c|c|c|c|c|}
\hline \multirow[t]{3}{*}{$\alpha=a / t$} & $b / a=1.0$ & $b / a=1.2$ & $b / a=1.4$ & $b / a=1.6$ & $b / a=1.8$ & $b / a=2.0$ & $b / a=2.2$ & $b / a=2.4$ \\
\hline & \multicolumn{8}{|c|}{$\lambda=\frac{\Delta}{a^{2}} \sqrt{\frac{D}{m}}$} \\
\hline & \multicolumn{8}{|c|}{$\Delta$} \\
\hline 5 & 17.8439 & 15.3339 & 13.7871 & 12.7692 & 12.0648 & 11.5576 & 11.1806 & 10.8928 \\
\hline 10 & 19.2148 & 16.3460 & 14.6052 & 13.4708 & 12.6911 & 12.1323 & 11.7184 & 11.4032 \\
\hline 15 & 19.5059 & 16.5569 & 14.7736 & 13.6142 & 12.8184 & 12.2487 & 11.8269 & 11.5060 \\
\hline 20 & 19.6110 & 16.6327 & 14.8340 & 13.6655 & 12.8638 & 12.2902 & 11.8656 & 11.5427 \\
\hline 25 & 19.6602 & 16.6681 & 14.8622 & 13.6894 & 12.8851 & 12.3096 & 11.8837 & 11.5597 \\
\hline 30 & 19.6872 & 16.6875 & 14.8776 & 13.7025 & 12.8966 & 12.3201 & 11.8935 & 11.5691 \\
\hline 40 & 19.7140 & 16.7068 & 14.8930 & 13.7155 & 12.9082 & 12.3307 & 11.9033 & 11.5783 \\
\hline 50 & 19.7265 & 16.7158 & 14.9001 & 13.7215 & 12.9135 & 12.3355 & 11.9079 & 11.5826 \\
\hline 60 & 19.7333 & 16.7206 & 14.9039 & 13.7248 & 12.9164 & 12.3382 & 11.9104 & 11.5850 \\
\hline 70 & 19.7374 & 16.7236 & 14.9063 & 13.7268 & 12.9182 & 12.3398 & 11.9119 & 11.5864 \\
\hline 80 & 19.7400 & 16.7255 & 14.9078 & 13.7281 & 12.9193 & 12.3408 & 11.9128 & 11.5873 \\
\hline 90 & 19.7419 & 16.7268 & 14.9088 & 13.7290 & 12.9201 & 12.3415 & 11.9135 & 11.5879 \\
\hline 100 & 19.7432 & 16.7277 & 14.9096 & 13.7296 & 12.9207 & 12.3421 & 11.9140 & 11.5884 \\
\hline
\end{tabular}

Table-1: Non-dimensional fundamental natural frequency parameter, $\boldsymbol{\Delta}$

\begin{tabular}{|c|c|c|c|c|}
\hline \multirow{3}{*}{$\begin{array}{l}P= \\
b / a\end{array}$} & \multirow{3}{*}{$\begin{array}{l}\alpha= \\
a / t\end{array}$} & Present Study, (P.S). & Hashemi and Arsanjani, (2004) [8] & \multirow{3}{*}{$\begin{array}{l}\text { \% Difference. }= \\
\frac{(P . S-H . A) * 100}{P . S}\end{array}$} \\
\hline & & \multirow{2}{*}{\multicolumn{2}{|c|}{$\lambda=\frac{\Delta}{a^{2}} \sqrt{\frac{D}{m}}$}} & \\
\hline & & & & \\
\hline \multirow[t]{5}{*}{1} & 100.00 & 19.7432 & 19.7322 & 0.06 \\
\hline & 20.00 & 19.6110 & 19.5676 & 0.22 \\
\hline & 10.00 & 19.2148 & 19.084 & 0.68 \\
\hline & 6.67 & 18.6065 & 18.3661 & 1.29 \\
\hline & 5.00 & 17.8439 & 17.5055 & 1.90 \\
\hline \multirow[t]{5}{*}{1.5} & 100.00 & 14.2607 & 14.2525 & 0.06 \\
\hline & 20.00 & 14.1915 & 14.1662 & 0.18 \\
\hline & 10.00 & 13.9818 & 13.9085 & 0.52 \\
\hline & 6.67 & 13.653 & 13.5147 & 1.01 \\
\hline & 5.00 & 13.2287 & 13.025 & 1.54 \\
\hline \multirow[t]{5}{*}{2} & 100.00 & 12.3421 & 12.3343 & 0.06 \\
\hline & 20.00 & 12.2902 & 12.2696 & 0.17 \\
\hline & 10.00 & 12.1323 & 12.0752 & 0.47 \\
\hline & 6.67 & 11.8829 & 11.7747 & 0.91 \\
\hline & 5.00 & 11.5576 & 11.3961 & 1.40 \\
\hline \multirow[t]{5}{*}{2.5} & 100.00 & 11.4541 & 11.4464 & 0.07 \\
\hline & 20.00 & 11.4094 & 11.3906 & 0.16 \\
\hline & 10.00 & 11.2731 & 11.226 & 0.42 \\
\hline & 6.67 & 11.057 & 10.9617 & 0.86 \\
\hline & 5.00 & 10.7738 & 10.6307 & 1.33 \\
\hline
\end{tabular}

Table-2: Present Study Compared with Hashemi and Arsanjani, (2004) [8] for SSSS thick Plates 


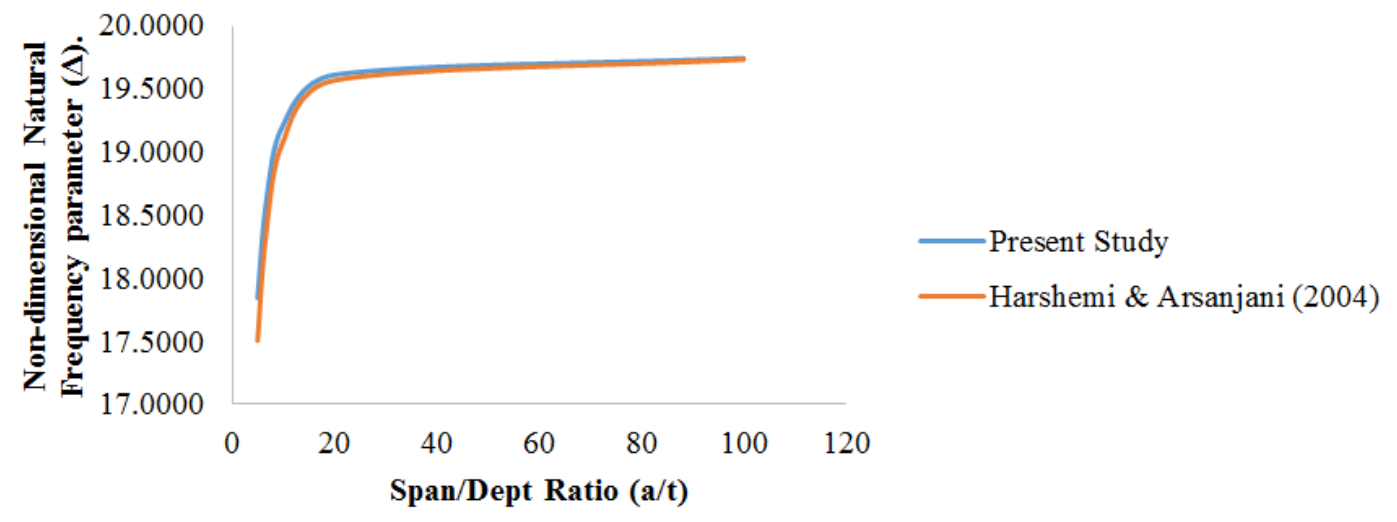

Fig-2: Present Study result compared with the Results of Harshemi \& Arsanjani (2004) [8]

Table-3: Present Study Compared with Rajesh and Meera (2016) [9] for SSSS thick Plates, at $P=b / a=2$

\begin{tabular}{|c|c|c|c|}
\hline \multirow{3}{*}{$\begin{array}{l}\alpha= \\
a / t\end{array}$} & Present Study (P.S) & Rajesh \& Meera (2016). (R.M) & \multirow{3}{*}{$\begin{array}{l}\% \text { Difference. }= \\
\frac{(\text { P.S }- \text { R.M }) * 100}{\text { P.S }}\end{array}$} \\
\hline & \multicolumn{2}{|r|}{$\lambda=\frac{\Delta}{a^{2}} \sqrt{\frac{D}{m}}$} & \\
\hline & \multicolumn{2}{|r|}{$\Delta$} & \\
\hline 5 & 11.5576 & 11.3716 & 1.61 \\
\hline 7 & 11.9232 & 11.8087 & 0.96 \\
\hline 9 & 12.0842 & 12.0073 & 0.64 \\
\hline 10 & 12.1323 & 12.0675 & 0.53 \\
\hline 11 & 12.1683 & 12.1127 & 0.46 \\
\hline 13 & 12.2175 & 12.1749 & 0.35 \\
\hline 15 & 12.2487 & 12.2145 & 0.28 \\
\hline 20 & 12.2902 & 12.2675 & 0.18 \\
\hline 25 & 12.3096 & 12.2924 & 0.14 \\
\hline 30 & 12.3201 & 12.306 & 0.11 \\
\hline 40 & 12.3307 & 12.3195 & 0.09 \\
\hline 50 & 12.3355 & 12.3258 & 0.08 \\
\hline 60 & 12.3382 & 12.3292 & 0.07 \\
\hline 70 & 12.3398 & 12.3313 & 0.07 \\
\hline 80 & 12.3408 & 12.3326 & 0.07 \\
\hline 90 & 12.3415 & 12.3335 & 0.06 \\
\hline 100 & 12.3421 & 12.3342 & 0.06 \\
\hline
\end{tabular}

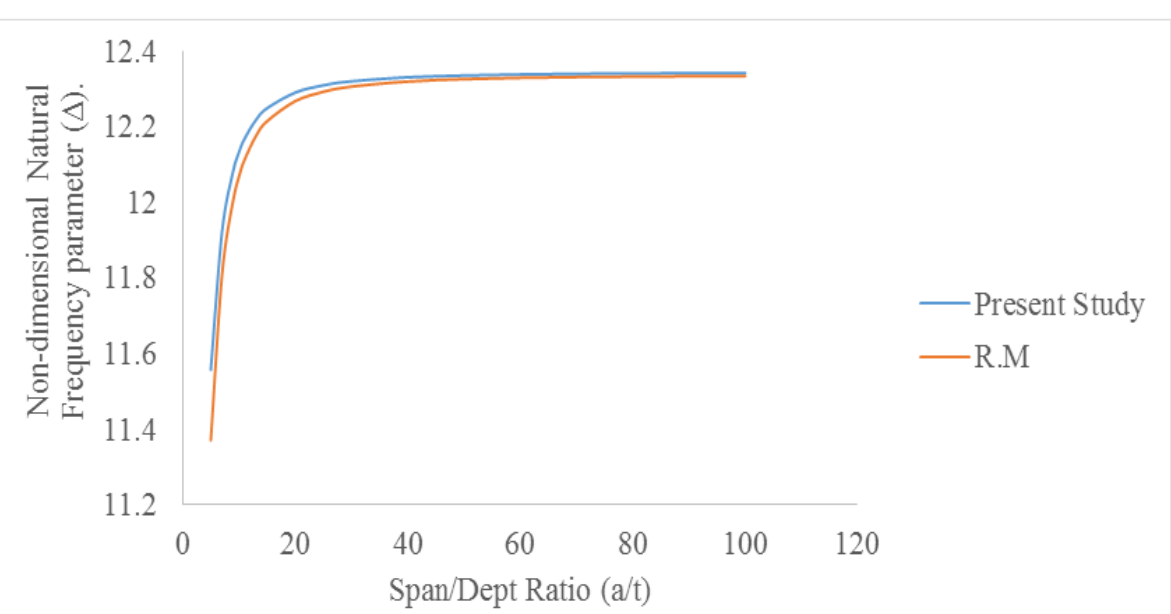

Fig-3: Present Study result compared with the Results of Rajesh and Meera (2016) [9] for aspect ratio, $P=2.0$ 
Looking at Table-2, at the same value of ' $P$ ', there is a decrease in the value of the non-dimensional natural frequency parameter as ' $a / t$ ' decreases. Also at any value of ' $a / t$ ', there is a decrease in the value of the non-dimensional natural frequency parameter $\Delta$ as ' $P$ ' increases, having the highest value at $P=1$ (square plate). Comparison between present study and the results of Hashemi and Arsanjani [8], shows a percentage difference range of (0.06 to 1.90). Figure-1 showed that the two results are close to each other. This confirms the efficacy of the present study.

It was observed from Table- 3 at the same value of ' $P$ ', there is an increase in the value of the nondimensional natural frequency parameter $\boldsymbol{\Delta}$ as ' $\mathrm{a} / \mathrm{t}$ ' increases. Comparing the present study with the results of Rajesh et al., [9], shows a percentage difference range of ( 0.06 to 1.90$)$. These differences are quite negligible and acceptable in statistics as being close. Also, Figure-2 showed that the two results are close to each other which attest to the efficacy of the present study.

\section{CONCLUSIONS}

The governing equation and the simple linear equation derived in this work produces speedy and acceptable results for free-vibration analysis of isotropic thick rectangular plates with simple supports at the four edges. The non-dimensional natural frequency parameters obtained in this work are close to the results of previous researchers and therefore are very reliable.

\section{REFERENCES}

1. Gorman, D. J. (1982). Free Vibration Analysis of Rectangular Plates. Elsevier North Holland Inc, USA.

2. Ezeh, J. C., Ibearugbulem, O. M., \& Onyechere I. C (2013). Pure bending analysis of thin rectangular flat plates using ordinary finite difference method. International Journal of Emerging Technology and Advanced Engineering. $3(3), 20-23$.

3. Ventsel, E., \& Krauthammer K. (2001). Thin Plates and Shells. New York, Marcel Decker Inc.

4. Sayyad, A. S., \& Ghugal Y. M. (2012). Bending and free vibration analysis of thick isotropic plates by using exponential shear deformation theory. Journal of Applied and Computational Mechanics. 6, 65-82.

5. Sayyad, I. I., Chikalthankar, S. B., \& Nandedkar, V. M. (2013). Bending and free vibration analysis of isotropic thick plate using refined plate theory. Bonfring International Journal of Industrial Engineering and Management Science. 3(2), 4046.

6. Sayyad, A. S. (2011). Comparison of various shear deformation theories for the free-vibration of thick isotropic beam. International Journal of Civil and Structural Engineering. 2(1), 85-97.

7. Sadrnejad, S. A., Daryan, A. S., \& Ziaei, M (2009). Vibration Equations of Thick Rectangular Plates Using Mindlin Plate Theory. Journal of Computer Science, 5(11), 838-842.

8. Hashemi, S. H., \& Arsanjani, M. (2004). Exact characteristic equations for some of classical boundary conditions of vibrating moderately thick rectangular plates. International Journal of Solid and Structures. 42, 819-853.

9. Rajesh, K., \& Meera, S. K. (2016). Linear free vibration analysis of rectangular Mindlin plates using coupled displacement field method. Mathematical Models in Engineering. 1(1), 41-47.

10. Onyechere, I. C. (2019). Stability and Vibration Analysis of Thick Plates using Orthogonal Polynomial Displacement Functions. PhD Thesis. Department of Civil Engineering, Federal University of Technology, Owerri (FUTO), Nigeria. 